\title{
An SOS-based Observer Design for Polynomial Fuzzy Systems
}

\author{
Kazuo Tanaka, Hiroshi Ohtake, Toshiaki Seo and Hua O. Wang
}

\begin{abstract}
This paper presents a sum of squares (SOS, for brevity) based observer design for a more general class of polynomial fuzzy systems with the polynomial matrices $\boldsymbol{A}_{i}(\boldsymbol{x}(t))$ and $\boldsymbol{B}_{i}(\boldsymbol{x}(t))$ that are permitted to be dependent of the states $\boldsymbol{x}(t)$. First, we briefly summarize previous works on SOS-based observer designs for two limited classes of polynomial fuzzy systems. To overcome the difficulty of the fact that does not realize the so-called separation principle design for the more general class, this paper provides a practical design procedure of a polynomial fuzzy controller and a polynomial fuzzy observer without lack of guaranteeing the stability of the overall control system in addition to converging state estimation error (via the observer) to zero. The design approach discussed in this paper is more general than the existing LMI approaches (to T-S fuzzy controller and observer designs) and also than the previous SOS-based observer designs. To illustrate the validity of the design approach, a design example is provided. The example shows the utility of our SOS approach to the polynomial fuzzy observer-based control for the more general class of polynomial fuzzy systems.
\end{abstract}

\section{INTRODUCTION}

The Takagi-Sugeno (T-S) fuzzy model-based control methodology [1] has received a great deal of attention after LMI-based designs have been discussed in [2]-[3]. The fuzzy model-based control methodology [1] provides a natural, simple and effective design approach to complement other nonlinear control techniques (e.g., [4]) that require special and rather involved knowledge.

Recently, the authors have first presented a sum of squares (SOS, for brevity) approach [5]-[10] to polynomial fuzzy control system designs. This is a completely different approach from the existing LMI approaches [1], [11]. Our SOS approach [5]-[10] provided more extensive results for the existing LMI approaches to T-S fuzzy model and control. After the SOS-based controller designs [5]-[10] have been addressed, we also presented an observer-based design [12] for the polynomial fuzzy systems with the polynomial matrices $\boldsymbol{A}_{i}$ and $\boldsymbol{B}_{i}$ that are independent of the states $\boldsymbol{x}$ to be estimated (shortly name it as Class I). Furthermore, we discussed an observer-based design [13] for a wider class of polynomial fuzzy systems with the polynomial matrices $\boldsymbol{A}_{i}$ that are permitted to be dependent of the states $\boldsymbol{x}$ to be estimated (shortly name it as Class II). Both of

This work was supported in part by a Grant-in-Aid for Scientific Research (C) 18560244 from the Ministry of Education, Science and Culture of Japan.

K. Tanaka, H. Ohtake, T. Seo are with the Department of Mechanical Engineering and Intelligent Systems, The University of Electro-Communications, Chofu, Tokyo 182-8585 Japan ktanaka@mce.uec.ac.jpl hohtake@mce.uec.ac.jp; toshiseo@rc.mce.uec.ac.jp

H. O. Wang is with the Department of Mechanical Engineering, Boston University, Boston, MA 02215 USA wangh@bu.edu the design conditions are represented in terms of SOS. More importantly, it should be emphasized that the so-called separation principle design realizes for both of the classes. This paper presents a polynomial fuzzy observer design for a more general class of polynomial fuzzy systems, i.e., the polynomial fuzzy systems with the polynomial matrices $\boldsymbol{A}_{i}$ and $\boldsymbol{B}_{i}$ that are permitted to be dependent of the states $\boldsymbol{x}$ to be estimated (shortly name it as Class III).

It is well known that stability conditions for the T-S fuzzy system reduce to LMIs, e.g., [1]. Hence, the stability conditions can be solved numerically and efficiently by interior point algorithms, e.g., by LMI solvers. On the other hand, some kinds of control design conditions [5]-[10] for polynomial fuzzy systems reduce to SOS problems. Clearly, the problem is never directly solved by LMI solvers and can be solved via the SOSTOOLS [14] and an SDP solver. Thus, SOS can be regarded as an extensive representation of LMIs. The computational method used in this paper relies on the SOS decomposition of multivariate polynomials. A multivariate polynomial $f\left(\boldsymbol{x}(t)\right.$ ) (where $\boldsymbol{x}(t) \in R^{n}$ ) is an SOS if there exist polynomials $f_{1}(\boldsymbol{x}(t)), \cdots, f_{k}(\boldsymbol{x}(t))$ such that $f(\boldsymbol{x}(t))=\sum_{i=1}^{k} f_{i}^{2}(\boldsymbol{x}(t))$. It is clear that $f(\boldsymbol{x}(t))$ being an SOS naturally implies $f(\boldsymbol{x}(t)) \geq 0$ for all $\boldsymbol{x}(t) \in R^{n}$. For more details of SOS, see [14].

\section{Polynomial FUZZY MODEL AND PREVIOUS RESUlTS}

Section II recalls a polynomial fuzzy system defined in [5][10] and summarizes the previous polynomial fuzzy observer design results [12], [13].

Consider the following nonlinear system:

$$
\dot{\boldsymbol{x}}(t)=f(\boldsymbol{x}(t), \boldsymbol{u}(t)),
$$

where $f$ is a nonlinear function. $\boldsymbol{x}(t)=$ $\left[\begin{array}{llll}x_{1}(t) & x_{2}(t) & \cdots & x_{n}(t)\end{array}\right]^{T}$ is the state vector and $\boldsymbol{u}(t)=\left[\begin{array}{llll}u_{1}(t) & u_{2}(t) & \cdots & u_{m}(t)\end{array}\right]^{T}$ is the input vector. In [5], we proposed a new type of fuzzy model with polynomial model consequence, i.e., fuzzy model whose consequent parts are represented by polynomials. Using the sector nonlinearity concept [1], we exactly represent (1) with the following polynomial fuzzy model (2). The main difference between the T-S fuzzy model [15] and the polynomial fuzzy model is consequent part representation. The fuzzy model (2) has a polynomial model consequence.

\section{Model Rule $i$ :}

$$
\begin{aligned}
& \text { If } z_{1}(t) \text { is } M_{i 1} \text { and } \cdots \text { and } z_{p}(t) \text { is } M_{i p} \\
& \text { then } \dot{\boldsymbol{x}}(t)=\boldsymbol{A}_{i}(\boldsymbol{x}(t)) \boldsymbol{x}(t)+\boldsymbol{B}_{i}(\boldsymbol{x}(t)) \boldsymbol{u}(t) \text {, }
\end{aligned}
$$


where $i=1,2, \cdots, r . r$ denotes the number of Model Rules. The membership function associated with the $i$ th Model Rule and the $j$ th premise variable component is denoted by $M_{i j} . z_{j}(t)(j=1,2, \cdots, p)$ is the premise variable. Each $z_{j}(t)$ is a measurable time-varying quantity that may be measurable external variables and/or time. Note that $z_{j}(t)$ is assumed to be independent of the states $\boldsymbol{x}$. $\boldsymbol{A}_{i}(\boldsymbol{x}(t)) \in \boldsymbol{R}^{n \times n}$ and $\boldsymbol{B}_{i}(\boldsymbol{x}(t)) \in \boldsymbol{R}^{n \times m}$ are polynomial matrices in $\boldsymbol{x}(t)$. Therefore, $\boldsymbol{A}_{i}(\boldsymbol{x}(t)) \boldsymbol{x}(t)+\boldsymbol{B}_{i}(\boldsymbol{x}(t)) \boldsymbol{u}(t)$ is a polynomial vector. Thus, the polynomial fuzzy model (2) has a polynomial in each consequent part.

The defuzzification process of the model (2) can be represented as

$$
\dot{\boldsymbol{x}}(t)=\sum_{i=1}^{r} h_{i}(\boldsymbol{z}(t))\left\{\boldsymbol{A}_{i}(\boldsymbol{x}(t)) \boldsymbol{x}(t)+\boldsymbol{B}_{i}(\boldsymbol{x}(t)) \boldsymbol{u}(t)\right\},
$$

where

$$
h_{i}(\boldsymbol{z}(t))=\frac{\prod_{j=1}^{p} M_{i j}\left(z_{j}(t)\right)}{\sum_{k=1}^{r} \prod_{j=1}^{p} M_{k j}\left(z_{j}(t)\right)} .
$$

It should be noted from the properties of membership functions that $h_{i}(\boldsymbol{z}(t)) \geq 0$ for all $i$ and $\sum_{i=1}^{r} h_{i}(\boldsymbol{z}(t))=1$. Thus, the overall fuzzy model is achieved by fuzzy blending of the polynomial system models.

Remark 1: As shown in [5]-[9], the number of rules in polynomial fuzzy model generally becomes fewer than that in T-S fuzzy model, and our SOS approach to polynomial fuzzy models provides much more relaxed results than the existing LMI approaches to T-S fuzzy model and control. The local polynomial systems in the consequent parts of (2) may be represented with monomial vectors in $\boldsymbol{x}(t)$ as defined in [5]-[9]. However, it should be noted that the local polynomial systems in the consequent parts of (2) are essentially equivalent representation of those defined in [5][9].

It is not simple to design a polynomial observer for (3) since $\boldsymbol{A}_{i}(\boldsymbol{x}(t))$ and $\boldsymbol{B}_{i}(\boldsymbol{x}(t))$ are dependent of the states $\boldsymbol{x}(t)$ to be estimated. As a first step, we introduce the following classes of polynomial fuzzy systems.

$\dot{\boldsymbol{x}}(t)=\sum_{i=1}^{r} h_{i}(\boldsymbol{z}(t))\left\{\boldsymbol{A}_{i}\left(\boldsymbol{\rho}_{A}(t)\right) \boldsymbol{x}(t)+\boldsymbol{B}_{i}\left(\boldsymbol{\rho}_{B}(t)\right) \boldsymbol{u}(t)\right\}$,

where (4) reduces to (3) when $\boldsymbol{\rho}_{A}(t)=\boldsymbol{\rho}_{B}(t)=\boldsymbol{x}(t)$.

In the previous papers [12], [13], we discussed polynomial observer-based designs for two classes:

Class I[12]: $\boldsymbol{\rho}_{A}(t)=\boldsymbol{y}(t)$ and $\boldsymbol{\rho}_{B}(t)=\boldsymbol{y}(t)$.

Class II[13]: $\boldsymbol{\rho}_{A}(t)=\boldsymbol{x}(t)$ and $\boldsymbol{\rho}_{B}(t)=\boldsymbol{y}(t)$.

In this paper, we will discuss the most general class (Class III), that is, $\boldsymbol{\rho}_{A}(t)=\boldsymbol{\rho}_{B}(t)=\boldsymbol{x}(t)$.

From now, to lighten the notation, we will drop the notation with respect to time $t$. For instance, we will employ $\boldsymbol{x}$ and $\hat{\boldsymbol{x}}$ instead of $\boldsymbol{x}(t)$ and $\hat{\boldsymbol{x}}(t)$, respectively, where $\hat{\boldsymbol{x}}(t)$ denotes the state estimated by polynomial fuzzy observers as will be discussed later. Thus, we drop the notation with respect to time $t$, but it should be kept in mind that $\boldsymbol{x}$ and $\hat{\boldsymbol{x}}$ means $\boldsymbol{x}(t)$ and $\hat{\boldsymbol{x}}(t)$, respectively.

Next, we define the outputs for the polynomial fuzzy model as

$$
\boldsymbol{y}=\sum_{i=1}^{r} h_{i}(\boldsymbol{z}) \boldsymbol{C}_{i} \boldsymbol{x}
$$

where $\boldsymbol{y} \in \boldsymbol{R}^{q}$ is the output.

\section{A. Class I}

Class I design deals with the polynomial fuzzy model (6) and (5).

$$
\dot{\boldsymbol{x}}=\sum_{i=1}^{r} h_{i}(\boldsymbol{z})\left\{\boldsymbol{A}_{i}(\boldsymbol{y}) \boldsymbol{x}+\boldsymbol{B}_{i}(\boldsymbol{y}) \boldsymbol{u}\right\} .
$$

Clearly, all the elements in the $\boldsymbol{A}_{i}(\boldsymbol{y})$ and $\boldsymbol{B}_{i}(\boldsymbol{y})$ matrices are measurable. A polynomial fuzzy observer [12] in Class I is described as

$$
\begin{aligned}
& \dot{\hat{\boldsymbol{x}}}=\sum_{i=1}^{r} h_{i}(\mathbf{z})\left\{\boldsymbol{A}_{i}(\boldsymbol{y}) \hat{\boldsymbol{x}}+\boldsymbol{B}_{i}(\boldsymbol{y}) \boldsymbol{u}+\boldsymbol{L}_{i}(\boldsymbol{y})(\boldsymbol{y}-\hat{\boldsymbol{y}})\right\}, \\
& \hat{\boldsymbol{y}}=\sum_{i=1}^{r} h_{i}(\mathbf{z}) \boldsymbol{C}_{i} \hat{\boldsymbol{x}},
\end{aligned}
$$

where $\hat{\boldsymbol{x}} \in \boldsymbol{R}^{n}$ is the state estimated via the observer. $\hat{\boldsymbol{y}} \in$ $\boldsymbol{R}^{q}$ is the output of the observer. $\boldsymbol{L}_{i}(\boldsymbol{y}) \in \boldsymbol{R}^{n \times q}$ for all $i$ are the polynomial observer gain matrices in $\boldsymbol{y}$ for each local polynomial observer.

To stabilize the system (5) - (8), we design a polynomial fuzzy controller with the state feedback estimated by the polynomial fuzzy observer (7) and (8).

$$
\boldsymbol{u}=-\sum_{i=1}^{r} h_{i}(\mathbf{z}) \boldsymbol{F}_{i}(\boldsymbol{y}) \hat{\boldsymbol{x}},
$$

where $\boldsymbol{F}_{i}(\boldsymbol{y})$ for all $i$ are the polynomial feedback gain matrices in $\boldsymbol{y}$. The SOS-based observer design discussed in [12] guarantees the so-called separation principle design for the overall control system consisting of the polynomial fuzzy system (6) and (5), the fuzzy controller (9) and the fuzzy observer (7) and (8).

Remark 2: If the polynomial matrices $\boldsymbol{A}_{i}, \boldsymbol{B}_{i}, \boldsymbol{L}_{i}$ and $\boldsymbol{F}_{i}$ reduce to constant matrices in (5) - (9), they reduce to the ordinary T-S fuzzy model [15], the T-S fuzzy controller [1] and the T-S fuzzy observer [16]. In addition, the SOS-based observer design discussed in [12] reduces to the existing LMI design conditions [16] for the T-S fuzzy controller and observers. Hence, the SOS-based observer design discussed in [12] provides more relaxed results.

\section{B. Class II}

Class II considers more complicated class, i.e., $\boldsymbol{A}_{i}$ depends on the state $\boldsymbol{x}$ instead of the output $\boldsymbol{y}$. Class II design deals with the polynomial fuzzy system (10) and (5)

$$
\dot{\boldsymbol{x}}=\sum_{i=1}^{r} h_{i}(\boldsymbol{z})\left\{\boldsymbol{A}_{i}(\boldsymbol{x}) \boldsymbol{x}+\boldsymbol{B}_{i}(\boldsymbol{y}) \boldsymbol{u}\right\}
$$


We design a polynomial fuzzy observer to estimate the states of (10) and (5).

$$
\begin{aligned}
& \dot{\hat{\boldsymbol{x}}}=\sum_{i=1}^{r} h_{i}(\boldsymbol{z})\left\{\boldsymbol{A}_{i}(\hat{\boldsymbol{x}}) \hat{\boldsymbol{x}}+\boldsymbol{B}_{i}(\boldsymbol{y}) \boldsymbol{u}+\boldsymbol{L}_{i}(\boldsymbol{y}, \hat{\boldsymbol{x}})(\boldsymbol{y}-\hat{\boldsymbol{y}})\right\} \\
& \hat{\boldsymbol{y}}=\sum_{i=1}^{r} h_{i}(\boldsymbol{z}) \boldsymbol{C}_{i} \hat{\boldsymbol{x}}
\end{aligned}
$$

where $\boldsymbol{L}_{i}(\boldsymbol{y}, \hat{\boldsymbol{x}})$ for all $i$ are the polynomial observer gain matrices in $\boldsymbol{y}$ and $\hat{\boldsymbol{x}}$. To stabilize the system (5) and (10) (12), we design a polynomial fuzzy controller with the state feedback estimated by the polynomial observer.

$$
\boldsymbol{u}=-\sum_{i=1}^{r} h_{i}(\boldsymbol{z}) \boldsymbol{F}_{i}(\hat{\boldsymbol{x}}) \hat{\boldsymbol{x}}
$$

where $\boldsymbol{F}_{i}(\hat{\boldsymbol{x}})$ for all $i$ are the polynomial feedback gain matrices in $\hat{\boldsymbol{x}}$. SOS design conditions to determine $\boldsymbol{F}_{i}(\hat{\boldsymbol{x}})$ and $\boldsymbol{L}_{i}(\boldsymbol{y}, \hat{\boldsymbol{x}})$ in Class II are derived in [13]. In general, even in this class, it is difficult to separately design the polynomial fuzzy controller (13) and observer (11) - (12). However, an important lemma to realize the separation principle design is presented in [13]. As a result, the SOS design conditions also realize the separation principle design as well as in Class I.

\section{Main Results:Class III Design}

This section presents main results of this paper, i.e., the most general class (Class III) design. Class III design deals with the polynomial fuzzy system (14) and (5).

$$
\dot{\boldsymbol{x}}=\sum_{i=1}^{r} h_{i}(\boldsymbol{z})\left\{\boldsymbol{A}_{i}(\boldsymbol{x}) \boldsymbol{x}+\boldsymbol{B}_{i}(\boldsymbol{x}) \boldsymbol{u}\right\}
$$

For the system (14) and (5), we design the following polynomial fuzzy observer.

$$
\begin{aligned}
& \dot{\hat{\boldsymbol{x}}}=\sum_{i=1}^{r} h_{i}(\boldsymbol{z})\left\{\boldsymbol{A}_{i}(\hat{\boldsymbol{x}}) \hat{\boldsymbol{x}}+\boldsymbol{B}_{i}(\hat{\boldsymbol{x}}) \boldsymbol{u}+\boldsymbol{L}_{i}(\boldsymbol{y}, \hat{\boldsymbol{x}})(\boldsymbol{y}-\hat{\boldsymbol{y}})\right. \\
& \hat{\boldsymbol{y}}=\sum_{i=1}^{r} h_{i}(\boldsymbol{z}) \boldsymbol{C}_{i} \hat{\boldsymbol{x}}
\end{aligned}
$$

where $\boldsymbol{L}_{i}(\boldsymbol{y}, \hat{\boldsymbol{x}})$ for all $i$ are the polynomial observer gain matrices in $\boldsymbol{y}$ and $\hat{\boldsymbol{x}}$. It is extremely difficult to separately design a polynomial fuzzy controller and observer in Class III. In fact, to the best of our knowledge, there exist no literatures on achieving the separation principle design in this class. To overcome the difficulty, we propose a practical algorithm to design a polynomial fuzzy controller and observer satisfying the stability of the overall augmented system in addition to converging state estimation error (via the observer) to zero.

The algorithm mainly consists of three steps.

Step 1 By assuming that all the states are measurable, we design the following controller.

$$
\boldsymbol{u}=-\sum_{i=1}^{r} h_{i}(\boldsymbol{z}) \boldsymbol{F}_{i}(\boldsymbol{x}) \boldsymbol{x}
$$

The SOS conditions (see Theorem 1 below) derived in [6], [8] are applied to determine the polynomial feedback gains $\boldsymbol{F}_{i}(\boldsymbol{x})$.

Step 2 We replace the controller designed in Step 1 with

$$
\boldsymbol{u}=-\sum_{i=1}^{r} h_{i}(\boldsymbol{z}) \boldsymbol{F}_{i}(\hat{\boldsymbol{x}}) \hat{\boldsymbol{x}}
$$

where $\boldsymbol{x}$ is replaced with $\hat{\boldsymbol{x}}$.

Step 3 Note that $\boldsymbol{F}_{i}(\hat{\boldsymbol{x}})$ obtained in Step 2 are known matrices. We determine the polynomial observer gains $\boldsymbol{L}_{i}(\boldsymbol{y}, \hat{\boldsymbol{x}})$ by solving new SOS design conditions (see Theorem 2 below).

We present the previous SOS conditions [6], [8] (Theorem 1 below) to determine the polynomial feedback gains $\boldsymbol{F}_{i}(\boldsymbol{x})$ and new SOS design conditions (Theorem 2 below) to determine the polynomial observer gains that are newly derived in this paper.

Theorem 1: [6], [8] The system (14) and (5) can be stabilized by the controller (17) if there exist a positive definite matrix $\boldsymbol{X}_{1} \in \mathbb{R}^{n \times n}$ and polynomial matrices $\boldsymbol{M}_{i}(\boldsymbol{x}) \in$ $\mathbb{R}^{p \times n}$ satisfying the following SOS conditions.

$$
\begin{aligned}
& \boldsymbol{v}_{1}^{T}\left(\boldsymbol{X}_{1}-\epsilon_{r_{1}} \boldsymbol{I}\right) \boldsymbol{v}_{1} \text { is } S O S \\
& -\boldsymbol{v}_{2}^{T}\left(\boldsymbol{X}_{1} \boldsymbol{A}_{i}^{T}(\boldsymbol{x})-\boldsymbol{M}_{i}(\boldsymbol{x}) \boldsymbol{B}_{i}^{T}(\boldsymbol{x})\right. \\
& \left.\quad+\boldsymbol{A}_{i}(\boldsymbol{x}) \boldsymbol{X}_{1}-\boldsymbol{B}_{i}(\boldsymbol{x}) \boldsymbol{M}_{i}(\boldsymbol{x})+\epsilon_{r_{2 i}}(\boldsymbol{x}) \boldsymbol{I}\right) \boldsymbol{v}_{2} \\
& \quad \text { is } O S \\
& -\boldsymbol{v}_{3}^{T}\left(\boldsymbol{X}_{1} \boldsymbol{A}_{i}^{T}(\boldsymbol{x})-\boldsymbol{M}_{j}(\boldsymbol{x}) \boldsymbol{B}_{i}^{T}(\boldsymbol{x})\right. \\
& \quad+\boldsymbol{A}_{i}(\boldsymbol{x}) \boldsymbol{X}_{1}-\boldsymbol{B}_{i}(\boldsymbol{x}) \boldsymbol{M}_{j}(\boldsymbol{x}) \\
& \quad+\boldsymbol{X}_{1} \boldsymbol{A}_{j}^{T}(\boldsymbol{x})-\boldsymbol{M}_{i}(\boldsymbol{x}) \boldsymbol{B}_{j}^{T}(\boldsymbol{x}) \\
& \left.\quad+\boldsymbol{A}_{j}(\boldsymbol{x}) \boldsymbol{X}_{1}-\boldsymbol{B}_{j}(\boldsymbol{x}) \boldsymbol{M}_{i}(\boldsymbol{x})+\epsilon_{r_{3 i j}}(\boldsymbol{x}) \boldsymbol{I}\right) \boldsymbol{v}_{3} \\
& \quad \text { is } \quad S O S \quad i<j
\end{aligned}
$$

where $\boldsymbol{v}_{1}, \boldsymbol{v}_{2}, \boldsymbol{v}_{3} \in \mathbb{R}^{n}$ denote vectors that are independent of $\boldsymbol{x} . \epsilon_{r_{1}}$ is a positive value. $\epsilon_{r_{2 i}}(\boldsymbol{x})$ and $\epsilon_{r_{3 i j}}(\boldsymbol{x})$ are nonnegative polynomials such that $\epsilon_{r_{2 i}}(\boldsymbol{x})>0$ and $\epsilon_{r_{3 i j}}(\boldsymbol{x})>0$ for $\boldsymbol{x} \neq \boldsymbol{0}$. From the solutions $\boldsymbol{X}_{1}$ and $\boldsymbol{M}_{i}(\boldsymbol{x})$, the feedback gain can be obtained as $\boldsymbol{F}_{i}(\boldsymbol{x})=\boldsymbol{M}_{i}(\boldsymbol{x}) \boldsymbol{X}_{1}^{-1}$.

Theorem 2: The system (14) and (5) can be stabilized by the polynomial fuzzy controller (18) and the estimation error via the polynomial fuzzy observer (15) and (16) tends to zero if there exist a positive definite matrix $\boldsymbol{X}_{2} \in \mathbb{R}^{n \times n}$ and polynomial matrices $\boldsymbol{N}(\boldsymbol{y}, \hat{\boldsymbol{x}}) \in \mathbb{R}^{n \times q}$ satisfying the following SOS conditions, where $\boldsymbol{X}_{1}$ and $\boldsymbol{F}_{j}(\hat{\boldsymbol{x}})$ are solutions satisfying the SOS conditions in Theorem 1 and are given 
(known) matrices in Theorem 2.

$$
\begin{aligned}
& \boldsymbol{x}_{v}^{T}\left(\left[\begin{array}{cc}
\boldsymbol{X}_{1}^{-1} \boldsymbol{X}_{2} & \mathbf{0} \\
\mathbf{0} & \boldsymbol{X}_{2}
\end{array}\right]-\epsilon_{o_{1}} \boldsymbol{I}\right) \boldsymbol{x}_{v} \text { is } S O S \\
& -\boldsymbol{x}_{v}^{T}\left(\left[\begin{array}{ll}
\boldsymbol{\Omega}_{i i}^{11} & \boldsymbol{\Omega}_{i i}^{12} \\
\boldsymbol{\Omega}_{i i}^{21} & \boldsymbol{\Omega}_{i i}^{22}
\end{array}\right]+\epsilon_{o_{2 i}}(\boldsymbol{x}, \boldsymbol{y}, \hat{\boldsymbol{x}}) \boldsymbol{I}\right) \boldsymbol{x}_{v} \text { is } S O S \\
& -\boldsymbol{x}_{v}^{T}\left(\left[\begin{array}{ll}
\boldsymbol{\Omega}_{i j}^{11}+\boldsymbol{\Omega}_{j i}^{11} & \boldsymbol{\Omega}_{i j}^{12}+\boldsymbol{\Omega}_{j i}^{12} \\
\boldsymbol{\Omega}_{i j}^{21}+\boldsymbol{\Omega}_{j i}^{21} & \boldsymbol{\Omega}_{i j}^{22}+\boldsymbol{\Omega}_{j i}^{22}
\end{array}\right]+\epsilon_{o_{3 i}}(\boldsymbol{x}, \boldsymbol{y}, \hat{\boldsymbol{x}}) \boldsymbol{I}\right) \boldsymbol{x}_{v}
\end{aligned}
$$

where

$$
\begin{aligned}
& \boldsymbol{\Omega}_{i j}^{11}=\boldsymbol{X}_{1}^{-1} \boldsymbol{X}_{2}\left(\boldsymbol{A}_{i}(\hat{\boldsymbol{x}})-\boldsymbol{B}_{i}(\hat{\boldsymbol{x}}) \boldsymbol{F}_{j}(\hat{\boldsymbol{x}})\right) \\
& \boldsymbol{\Omega}_{i j}^{12}=\boldsymbol{X}_{1}^{-1} \boldsymbol{N}_{i}(\boldsymbol{y}, \hat{\boldsymbol{x}}) \boldsymbol{C}_{j} \\
& \boldsymbol{\Omega}_{i j}^{21}=\boldsymbol{X}_{2}\left(\boldsymbol{A}_{i}(\boldsymbol{x})-\boldsymbol{A}_{i}(\hat{\boldsymbol{x}})\right. \\
& \left.\quad-\left(\boldsymbol{B}_{i}(\boldsymbol{x})-\boldsymbol{B}_{i}(\hat{\boldsymbol{x}})\right) \boldsymbol{F}_{j}(\hat{\boldsymbol{x}})\right) \\
& \boldsymbol{\Omega}_{i j}^{22}=\boldsymbol{X}_{2} \boldsymbol{A}_{i}(\boldsymbol{x})-\boldsymbol{N}_{i}(\boldsymbol{y}, \hat{\boldsymbol{x}}) \boldsymbol{C}_{j}
\end{aligned}
$$

$\boldsymbol{x}_{v}=\left[\begin{array}{ll}\hat{\boldsymbol{x}} & \boldsymbol{e}\end{array}\right]^{T}, \boldsymbol{e}=\boldsymbol{x}-\hat{\boldsymbol{x}} . \epsilon_{O_{1}}$ is a positive value. $\epsilon_{O_{2 i}}(\boldsymbol{x}, \boldsymbol{y}, \hat{\boldsymbol{x}})$ and $\epsilon_{O_{3 i j}}(\boldsymbol{x}, \boldsymbol{y}, \hat{\boldsymbol{x}})$ are nonnegative polynomials such that $\epsilon_{O_{2 i}}(\boldsymbol{x}, \boldsymbol{y}, \hat{\boldsymbol{x}})>0$ and $\epsilon_{o_{3 i j}}(\boldsymbol{x}, \boldsymbol{y}, \hat{\boldsymbol{x}})>0$ for $\boldsymbol{x}, \boldsymbol{y}, \hat{\boldsymbol{x}} \neq \mathbf{0}$. From the solutions $\boldsymbol{X}_{2}$ and $\boldsymbol{N}_{i}(\boldsymbol{y}, \hat{\boldsymbol{x}})$, we can obtain observer gain matrices as $\boldsymbol{L}_{i}(\boldsymbol{y}, \hat{\boldsymbol{x}})=\boldsymbol{X}_{2}^{-1} \boldsymbol{N}_{i}(\boldsymbol{y}, \hat{\boldsymbol{x}})$.

Proof: Define the estimation error via the observer as $\boldsymbol{e}=\boldsymbol{x}-\hat{\boldsymbol{x}}$. Then, the error dynamics are represented as

$$
\begin{aligned}
& \dot{e}= \sum_{i=1}^{r} \sum_{j=1}^{r} h_{i}(\boldsymbol{z}) h_{j}(\boldsymbol{z}) \\
&\left\{\left(\boldsymbol{A}_{i}(\boldsymbol{x})-\boldsymbol{A}_{i}(\hat{\boldsymbol{x}})-\left(\boldsymbol{B}_{i}(\boldsymbol{x})-\boldsymbol{B}_{i}(\hat{\boldsymbol{x}})\right) \boldsymbol{F}_{j}(\hat{\boldsymbol{x}})\right) \hat{\boldsymbol{x}}\right. \\
&\left.\quad+\left(\boldsymbol{A}_{i}(\boldsymbol{x})-\boldsymbol{L}_{i}(\boldsymbol{y}, \hat{\boldsymbol{x}}) \boldsymbol{C}_{j}\right) \boldsymbol{e}\right\} .
\end{aligned}
$$

Next, consider an augmented system with the vector

$$
\boldsymbol{x}_{v}=\left[\begin{array}{l}
\hat{\boldsymbol{x}} \\
\boldsymbol{e}
\end{array}\right] \text {. }
$$

We obtain the following augmented system:

$$
\begin{aligned}
& \dot{\boldsymbol{x}}_{v}=\sum_{i=1}^{r} \sum_{j=1}^{r} h_{i}(\boldsymbol{z}) h_{j}(\boldsymbol{z}) \\
& {\left[\begin{array}{cc}
\boldsymbol{A}_{i}(\hat{\boldsymbol{x}})-\boldsymbol{B}_{i}(\hat{\boldsymbol{x}}) \boldsymbol{F}_{j}(\hat{\boldsymbol{x}}) & \boldsymbol{L}_{i}(\boldsymbol{y}, \hat{\boldsymbol{x}}) \boldsymbol{C}_{j} \\
\boldsymbol{A}_{i}(\boldsymbol{x})-\boldsymbol{A}_{i}(\hat{\boldsymbol{x}}) & \boldsymbol{A}_{i}(\boldsymbol{x})-\boldsymbol{L}_{i}(\boldsymbol{y}, \hat{\boldsymbol{x}}) \boldsymbol{C}_{j}
\end{array}\right] \boldsymbol{x}_{v}} \\
& -\left(\boldsymbol{B}_{i}(\boldsymbol{x})-\boldsymbol{B}_{i}(\hat{\boldsymbol{x}})\right) \boldsymbol{F}_{j}(\hat{\boldsymbol{x}}) \\
& =\sum_{i=1}^{r} \sum_{j=1}^{r} h_{i}(\boldsymbol{z}) h_{j}(\boldsymbol{z}) \boldsymbol{G}_{i j}(\boldsymbol{x}, \boldsymbol{y}, \hat{\boldsymbol{x}}) \boldsymbol{x}_{v},
\end{aligned}
$$

where

$$
\begin{aligned}
& \boldsymbol{G}_{i j}(\boldsymbol{x}, \boldsymbol{y}, \hat{\boldsymbol{x}})=\left[\begin{array}{cc}
\boldsymbol{G}_{i j}^{11}(\hat{\boldsymbol{x}}) & \boldsymbol{G}_{i j}^{12}(\boldsymbol{y}, \hat{\boldsymbol{x}}) \\
\boldsymbol{G}_{i j}^{21}(\boldsymbol{x}, \hat{\boldsymbol{x}}) & \boldsymbol{G}_{i j}^{22}(\boldsymbol{x}, \boldsymbol{y}, \hat{\boldsymbol{x}})
\end{array}\right] \\
& \boldsymbol{G}_{i j}^{11}(\hat{\boldsymbol{x}})=\boldsymbol{A}_{i}(\hat{\boldsymbol{x}})-\boldsymbol{B}_{i}(\hat{\boldsymbol{x}}) \boldsymbol{F}_{j}(\hat{\boldsymbol{x}}), \\
& \boldsymbol{G}_{i j}^{12}(\boldsymbol{y}, \hat{\boldsymbol{x}})=\boldsymbol{L}_{i}(\boldsymbol{y}, \hat{\boldsymbol{x}}) \boldsymbol{C}_{j}, \\
& \boldsymbol{G}_{i j}^{21}(\boldsymbol{x}, \hat{\boldsymbol{x}})=\boldsymbol{A}_{i}(\boldsymbol{x})-\boldsymbol{A}_{i}(\hat{\boldsymbol{x}})-\left(\boldsymbol{B}_{i}(\boldsymbol{x})-\boldsymbol{B}_{i}(\hat{\boldsymbol{x}})\right) \boldsymbol{F}_{j}(\hat{\boldsymbol{x}}), \\
& \boldsymbol{G}_{i j}^{22}(\boldsymbol{x}, \boldsymbol{y}, \hat{\boldsymbol{x}})=\boldsymbol{A}_{i}(\boldsymbol{x})-\boldsymbol{L}_{i}(\boldsymbol{y}, \hat{\boldsymbol{x}}) \boldsymbol{C}_{j} .
\end{aligned}
$$

Now, consider the following candidate of Lyapunov functions.

$$
V\left(\boldsymbol{x}_{v}\right)=\boldsymbol{x}_{v}^{T} \tilde{\boldsymbol{X}} \boldsymbol{x}_{v}
$$

where

$$
\tilde{\boldsymbol{X}}=\left[\begin{array}{cc}
\boldsymbol{X}_{1}^{-1} \boldsymbol{X}_{2} & \mathbf{0} \\
\mathbf{0} & \boldsymbol{X}_{2}
\end{array}\right]>\mathbf{0}
$$

The time derivative of $V\left(\boldsymbol{x}_{v}\right)$ along the system trajectories is

$$
\begin{aligned}
\dot{V}\left(\boldsymbol{x}_{v}\right)= & \sum_{i=1}^{r} \sum_{j=1}^{r} h_{i}(\boldsymbol{z}) h_{j}(\boldsymbol{z}) \boldsymbol{x}_{v}^{T}\left(\boldsymbol{G}_{i j}^{T}(\boldsymbol{x}, \boldsymbol{y}, \hat{\boldsymbol{x}}) \tilde{\boldsymbol{X}}\right. \\
& \left.+\tilde{\boldsymbol{X}} \boldsymbol{G}_{i j}(\boldsymbol{x}, \boldsymbol{y}, \hat{\boldsymbol{x}})\right) \boldsymbol{x}_{v} .
\end{aligned}
$$

Since $\boldsymbol{x}_{v}^{T} \boldsymbol{H} \boldsymbol{x}_{v}=\boldsymbol{x}_{v}^{T} \boldsymbol{H}^{T} \boldsymbol{x}_{v}$ for any square matrix $\boldsymbol{H}$, we have

$$
\begin{aligned}
\dot{V}\left(\boldsymbol{x}_{v}\right)= & 2 \sum_{i=1}^{r} \sum_{j=1}^{r} h_{i}(\boldsymbol{z}) h_{j}(\boldsymbol{z}) \boldsymbol{x}_{v}^{T} \tilde{\boldsymbol{X}} \boldsymbol{G}_{i j}(\boldsymbol{x}, \boldsymbol{y}, \hat{\boldsymbol{x}}) \boldsymbol{x}_{v} \\
= & 2 \sum_{i=1}^{r} h_{i}^{2}(\boldsymbol{z}) \boldsymbol{x}_{v}^{T} \tilde{\boldsymbol{X}} \boldsymbol{G}_{i i}(\boldsymbol{x}, \boldsymbol{y}, \hat{\boldsymbol{x}}) \boldsymbol{x}_{v} \\
& +2 \sum_{i=1}^{r} \sum_{i<j}^{r} h_{i}(\boldsymbol{z}) h_{j}(\boldsymbol{z}) \boldsymbol{x}_{v}^{T} \tilde{\boldsymbol{X}}\left(\boldsymbol{G}_{i j}(\boldsymbol{x}, \boldsymbol{y}, \hat{\boldsymbol{x}})\right. \\
& \left.+\boldsymbol{G}_{j i}(\boldsymbol{x}, \boldsymbol{y}, \hat{\boldsymbol{x}})\right) \boldsymbol{x}_{v} .
\end{aligned}
$$

$\dot{V}\left(\boldsymbol{x}_{v}\right)<0$ at $\boldsymbol{x}_{v} \neq \mathbf{0}$ if (31)-(33) hold.

$$
\begin{aligned}
& \boldsymbol{x}_{v}^{T} \tilde{\boldsymbol{X}} \boldsymbol{x}_{v}>\mathbf{0}, \\
& -\boldsymbol{x}_{v}^{T} \tilde{\boldsymbol{X}} \boldsymbol{G}_{i i}(\boldsymbol{x}, \boldsymbol{y}, \hat{\boldsymbol{x}}) \boldsymbol{x}_{v}>\mathbf{0}, \\
& -\boldsymbol{x}_{v}^{T} \tilde{\boldsymbol{X}}\left(\boldsymbol{G}_{i j}(\boldsymbol{x}, \boldsymbol{y}, \hat{\boldsymbol{x}})+\boldsymbol{G}_{j i}(\boldsymbol{x}, \boldsymbol{y}, \hat{\boldsymbol{x}})\right) \boldsymbol{x}_{v}>\mathbf{0} i<j .
\end{aligned}
$$

By defining as $\boldsymbol{N}_{i}(\boldsymbol{y}, \hat{\boldsymbol{x}})=\boldsymbol{X}_{2} \boldsymbol{L}_{i}(\boldsymbol{y}, \hat{\boldsymbol{x}})$, (32) can be rewritten as

$$
\begin{aligned}
\boldsymbol{x}_{v}^{T} \tilde{\boldsymbol{X}} \boldsymbol{G}_{i i}(\boldsymbol{x}, \boldsymbol{y}, \hat{\boldsymbol{x}}) \boldsymbol{x}_{v} & =\boldsymbol{x}_{v}^{T}\left[\begin{array}{cc}
\boldsymbol{\Omega}_{i i}^{11}(\hat{\boldsymbol{x}}) & \boldsymbol{\Omega}_{i i}^{12}(\boldsymbol{y}, \hat{\boldsymbol{x}}) \\
\boldsymbol{\Omega}_{i i}^{21}(\boldsymbol{x}, \hat{\boldsymbol{x}}) & \boldsymbol{\Omega}_{i i}^{22}(\boldsymbol{x}, \boldsymbol{y}, \hat{\boldsymbol{x}})
\end{array}\right] \boldsymbol{x}_{v}, \\
& =\boldsymbol{x}_{v}^{T} \boldsymbol{\Omega}_{i i}(\boldsymbol{x}, \boldsymbol{y}, \hat{\boldsymbol{x}}) \boldsymbol{x}_{v}
\end{aligned}
$$

where

$$
\begin{aligned}
& \boldsymbol{\Omega}_{i i}^{11}(\hat{\boldsymbol{x}})=\boldsymbol{X}_{1}^{-1} \boldsymbol{X}_{2}\left(\boldsymbol{A}_{i}(\hat{\boldsymbol{x}})-\boldsymbol{B}_{i}(\hat{\boldsymbol{x}}) \boldsymbol{F}_{j}(\hat{\boldsymbol{x}})\right), \\
& \boldsymbol{\Omega}_{i i}^{12}(\boldsymbol{y}, \hat{\boldsymbol{x}})=\boldsymbol{X}_{1}^{-1} \boldsymbol{N}_{i}(\boldsymbol{y}, \hat{\boldsymbol{x}}) \boldsymbol{C}_{j}, \\
& \boldsymbol{\Omega}_{i i}^{21}(\boldsymbol{x}, \hat{\boldsymbol{x}})=\boldsymbol{X}_{2}\left(\boldsymbol{A}_{i}(\boldsymbol{x})-\boldsymbol{A}_{i}(\hat{\boldsymbol{x}})\right. \\
& \left.-\left(\boldsymbol{B}_{i}(\boldsymbol{x})-\boldsymbol{B}_{i}(\hat{\boldsymbol{x}})\right) \boldsymbol{F}_{j}(\hat{\boldsymbol{x}})\right), \\
& \boldsymbol{\Omega}_{i i}^{22}(\boldsymbol{x}, \boldsymbol{y}, \hat{\boldsymbol{x}})=\boldsymbol{X}_{2} \boldsymbol{A}_{i}(\boldsymbol{x})-\boldsymbol{N}_{i}(\boldsymbol{y}, \hat{\boldsymbol{x}}) \boldsymbol{C}_{j} .
\end{aligned}
$$

Also, (33) can be rewritten as

$$
-\boldsymbol{x}_{v}^{T}\left(\boldsymbol{\Omega}_{i j}(\boldsymbol{x}, \boldsymbol{y}, \hat{\boldsymbol{x}})+\boldsymbol{\Omega}_{j i}(\boldsymbol{x}, \boldsymbol{y}, \hat{\boldsymbol{x}})\right) \boldsymbol{x}_{v}>\mathbf{0} \quad i<j
$$

where

$$
\begin{aligned}
\boldsymbol{\Omega}_{i j}^{11}(\hat{\boldsymbol{x}})=\boldsymbol{X}_{1}^{-1} \boldsymbol{X}_{2}\left(\boldsymbol{A}_{i}(\hat{\boldsymbol{x}})-\boldsymbol{B}_{i}(\hat{\boldsymbol{x}}) \boldsymbol{F}_{j}(\hat{\boldsymbol{x}})\right), \\
\boldsymbol{\Omega}_{i j}^{12}(\boldsymbol{y}, \hat{\boldsymbol{x}})=\boldsymbol{X}_{1}^{-1} \boldsymbol{N}_{i}(\boldsymbol{y}, \hat{\boldsymbol{x}}) \boldsymbol{C}_{j}, \\
\boldsymbol{\Omega}_{i j}^{21}(\boldsymbol{x}, \hat{\boldsymbol{x}})=\boldsymbol{X}_{2}\left(\boldsymbol{A}_{i}(\boldsymbol{x})-\boldsymbol{A}_{i}(\hat{\boldsymbol{x}})\right. \\
\left.\quad-\left(\boldsymbol{B}_{i}(\boldsymbol{x})-\boldsymbol{B}_{i}(\hat{\boldsymbol{x}})\right) \boldsymbol{F}_{j}(\hat{\boldsymbol{x}})\right), \\
\boldsymbol{\Omega}_{i j}^{22}(\boldsymbol{x}, \boldsymbol{y}, \hat{\boldsymbol{x}})=\boldsymbol{X}_{2} \boldsymbol{A}_{i}(\boldsymbol{x})-\boldsymbol{N}_{i}(\boldsymbol{y}, \hat{\boldsymbol{x}}) \boldsymbol{C}_{j} .
\end{aligned}
$$


Now, we arrive at the following SOSPs.

$$
\begin{gathered}
\boldsymbol{x}_{v}^{T}\left(\left[\begin{array}{cc}
\boldsymbol{X}_{1}^{-1} \boldsymbol{X}_{2} & \mathbf{0} \\
\mathbf{0} & \boldsymbol{X}_{2}
\end{array}\right]-\epsilon_{o_{1}} \boldsymbol{I}\right) \boldsymbol{x}_{v} \text { is } S O S \\
-\boldsymbol{x}_{v}^{T}\left(\boldsymbol{\Omega}_{i i}(\boldsymbol{x}, \boldsymbol{y}, \hat{\boldsymbol{x}})+\epsilon_{O_{2 i}}(\boldsymbol{x}, \boldsymbol{y}, \hat{\boldsymbol{x}}) \boldsymbol{I}\right) \boldsymbol{x}_{v} \text { is } S O S \\
-\boldsymbol{x}_{v}^{T}\left(\boldsymbol{\Omega}_{i j}(\boldsymbol{x}, \boldsymbol{y}, \hat{\boldsymbol{x}})+\boldsymbol{\Omega}_{j i}(\boldsymbol{x}, \boldsymbol{y}, \hat{\boldsymbol{x}})+\epsilon_{O_{3 i}}(\boldsymbol{x}, \boldsymbol{y}, \hat{\boldsymbol{x}}) \boldsymbol{I}\right) \boldsymbol{x}_{v} \\
i s \quad S O S \quad i<j
\end{gathered}
$$

where $\epsilon_{O_{1}}, \epsilon_{O_{2 i}}(\boldsymbol{x}, \boldsymbol{y}, \hat{\boldsymbol{x}}), \epsilon_{O_{3_{i j}}}(\boldsymbol{x}, \boldsymbol{y}, \hat{\boldsymbol{x}})$ are slack variables to keep the positivity of (31), (32) and (33).

Clearly, the overall control system consisting of (14), (5), (18), (15), and (16) are asymptotically and globally stable and the estimation error tends to zero.

Remark 3: Currently, sum of squares programs (SOSPs) are solved by reformulating them as semidefinite programs (SDPs). SOSTOOLS automates the conversion from SOSP to SDP and the SDP can be solved by a SDP solver [14]. At present, SOOSTOOLS uses other free MATLAB add-ons such as SeDuMi [17] or SDPT3 [18] as the SDP solver. In this paper, we numerically find $\boldsymbol{X}, \boldsymbol{M}_{i}(\boldsymbol{x})$ and $\boldsymbol{N}_{i}(\boldsymbol{x})$ satisfying the SOS conditions in Theorems 1 and 2 via SeDuMi in addition to SOSTOOLS. For more details of how to solve the SDPs using SeDuMi, see [14] and [17].

\section{DESIGN EXAMPLE}

Consider the following nonlinear system.

$$
\left\{\begin{array}{l}
\dot{x}_{1}=\sin x_{1}-5 x_{2}+\left(x_{2}^{2}+5\right) u \\
\dot{x}_{2}=-x_{1}-x_{2}^{3}
\end{array}\right.
$$

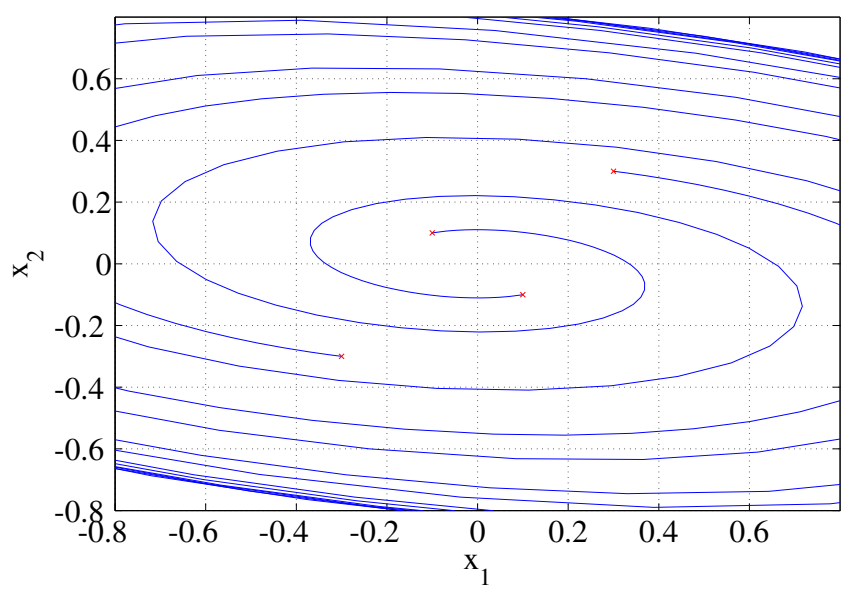

Fig. 1. System behavior without input.

Assume that $x_{1}$ is measurable and $y=x_{1}$. Fig. 1 shows the behavior of the nonlinear system without input for several initial states. It is found from the figure that this system is unstable.
The system (39) can be exactly converted into the polynomial fuzzy system (14) and (5) using the sector nonlinearity [1], where $r=2, \boldsymbol{z}=y$,

$$
\begin{aligned}
& \boldsymbol{A}_{1}(\boldsymbol{x})=\left[\begin{array}{cc}
1 & 5 \\
-1 & -x_{2}^{2}
\end{array}\right], \\
& \boldsymbol{A}_{2}(\boldsymbol{x})=\left[\begin{array}{cc}
-0.2172 & 5 \\
-1 & -x_{2}^{2}
\end{array}\right], \\
& \boldsymbol{B}_{1}(\boldsymbol{x})=\left[\begin{array}{c}
x_{2}^{2}+5 \\
0
\end{array}\right], \\
& \boldsymbol{B}_{2}(\boldsymbol{x})=\left[\begin{array}{c}
x_{2}^{2}+5 \\
0
\end{array}\right], \\
& \boldsymbol{C}_{1}=\boldsymbol{C}_{2}=\left[\begin{array}{ll}
1 & 0
\end{array}\right], \\
& h_{1}(\boldsymbol{z})=\frac{\sin y+0.2172 y}{1.2172 y}, \\
& h_{2}(\boldsymbol{z})=\frac{y-\sin y}{1.2172 y} .
\end{aligned}
$$

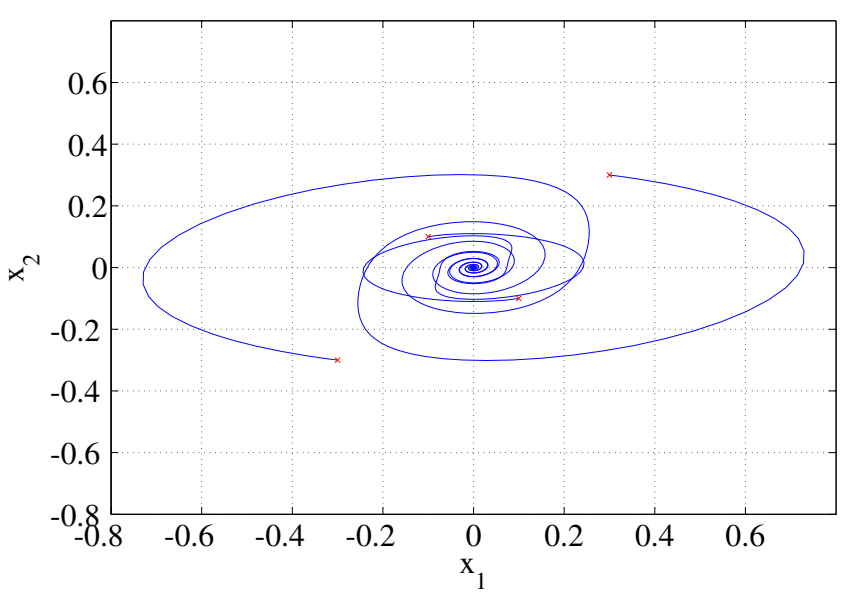

Fig. 2. Control trajectories for same initial states as in Fig. 1.

Fig. 2 shows control result (for the same initial states as Fig. 1) by the polynomial fuzzy controller and observer designed using Theorem 1 and Theorem 2, where the order of $\boldsymbol{M}_{i}(\hat{\boldsymbol{x}})$ and $\boldsymbol{N}_{i}(\boldsymbol{y}, \hat{\boldsymbol{x}})$ are two. Fig. 3 shows the control and estimation result starting from one of the initial states, where $\boldsymbol{x}(0)=\left[\begin{array}{ll}0.3 & 0.3\end{array}\right]$ and $\hat{\boldsymbol{x}}(0)=\left[\begin{array}{ll}-0.3 & -0.3\end{array}\right]$.

It can be found from the control results that the designed polynomial fuzzy controller stabilizes the system and the estimation error via the polynomial fuzzy observer tends to zero.

Remark 4: Since $\boldsymbol{A}_{1}(\boldsymbol{x}), \boldsymbol{A}_{2}(\boldsymbol{x}), \boldsymbol{B}_{1}(\boldsymbol{x})$ and $\boldsymbol{B}_{2}(\boldsymbol{x})$ have unmeasurable $x_{2}$ in this design example, the previous SOSbased observer designs discussed in [12] and [13] can not be applied to this design example. Even if the sector nonlinearity concept is applied to construct a Takagi-Sugeno fuzzy model for the nonlinear system, the premise variables $z$ contain $x_{2}$. Hence, the previous LMI conditions [16] can not be also applied to the nonlinear system. On the other hand, since our approach (Class III) discussed in this paper permits to 
have unmeasurable states in both of $\boldsymbol{A}_{i}$ and $\boldsymbol{B}_{i}$ matrices, it is possible to design a polynomial fuzzy observer in this example.

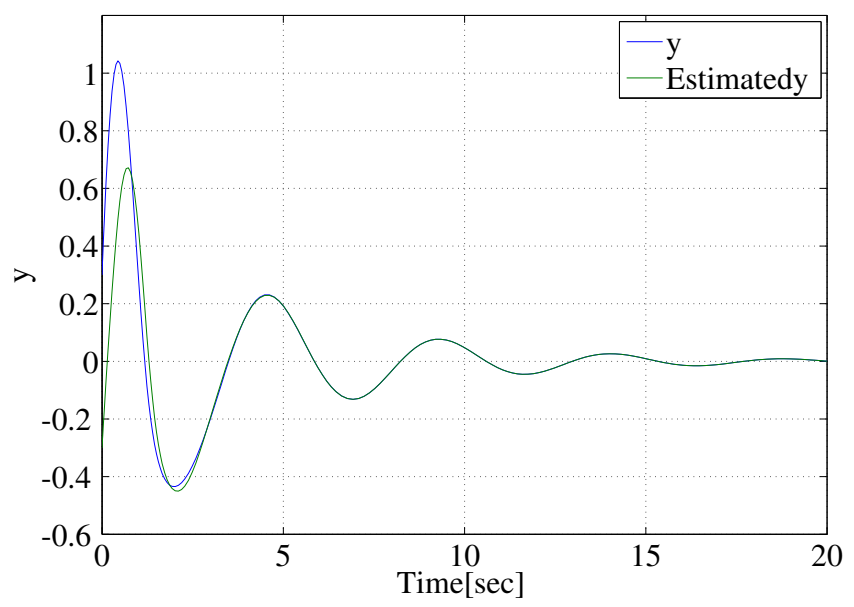

Fig. 3. Control and estimation result.

\section{CONCLUSiOnS}

This paper has presented a sum of squares (SOS) approach to polynomial fuzzy controller and observer designs for the more general class of polynomial fuzzy systems. To illustrate the validity and applicability of the proposed approach, a design example has been provided.

Our next subjects are to derive SOS-based observer design conditions to realize the separation principle design even for Class III and to apply our SOS-based observer designs to helicopter control [10].

\section{ACKNOWLEDGMENTS}

The authors would like to thank Mr. M. Wada, UEC, Japan, for his contribution to this research.

\section{REFERENCES}

[1] K. Tanaka and H. O. Wang: Fuzzy Control Systems Design and Analysis: A Linear Matrix Inequality Approach, JOHN WILEY \& SONS, INC, 2001

[2] H. O. Wang, K. Tanaka and M. F. Griffin, An Analytical Framework of Fuzzy Modeling and Control of Nonlinear Systems, 1995 American Control Conference, Seattle, Vol.3, pp.2272 - 2276 (1995).

[3] H. O. Wang, K. Tanaka and M. F. Griffin, An Approach to Fuzzy Control of Nonlinear Systems, IEEE Transactions on Fuzzy Systems, Vol.4, No.1, pp.14-23 (1996).

[4] R. Sepulcher, M. Jankovic and P. Kokotovic: Constructive Nonlinear Control, Springer, 1997

[5] K. Tanaka, H. Yoshida, H. Ohtake and H. O. Wang "A Sum of Squares Approach to Stability Analysis of Polynomial Fuzzy Systems”, 2007 American Control Conference, New York, July, 2007, pp.4071-4076.

[6] K. Tanaka, H. Yoshida, H. Ohtake and H. O. Wang, "Stabilization of Polynomial Fuzzy Systems via a Sum of Squares Approach”, 2007 IEEE International Symposium on Intelligent Control, pp.160-165, Singapore, October 2007.

[7] K. Tanaka, K. Yamauchi, H. Ohtake and H. O. Wang, Guaranteed Cost Control of Polynomial Fuzzy Systems via a Sum of Squares Approach, 2007 IEEE International Conference on Decision and Control, pp. 5954-5959, New Orleans, Dec. (2007)
[8] K. Tanaka, H. Yoshida, H. Ohtake and H. O. Wang, A Sum of Squares Approach to Modeling and Control of Nonlinear Dynamical Systems with Polynomial Fuzzy Systems, IEEE Transactions on Fuzzy Systems, Vol.17, No.4, pp.911-922, August 2009.

[9] K. Tanaka, H. Ohtake and H. O. Wang, Guaranteed Cost Control of Polynomial Fuzzy Systems via a Sum of Squares Approach, IEEE Transactions on Systems, Man and Cybernetics Part B, Vol.39, No.2, pp.561-567 April, 2009.

[10] K. Tanaka, T. Komatsu, H. Ohtake and H. O. Wang, Micro Helicopter Control:LMI Approach vs SOS Approach, 2008 IEEE International Conference on Fuzzy Systems, pp. 347-353, Hong Kong, June (2008)

[11] G. Feng, "A Survey on Analysis and Design of Model-Based Fuzzy Control Systems", IEEE Trans. on Fuzzy Systems, Vol.14, no.5, pp.676-697, Oct. 2006.

[12] K. Tanaka, H. Ohtake, M. Wada, H. O. Wang, Y.-J. Chen, Polynomial Fuzzy Observer Design: A Sum of Squares Approach, 48th IEEE conference on Decision and Control, pp. 7771 - 7776, Shanghai, China, December 16-18, 2009.

[13] T. Seo, H. Ohtake,Y.-J. Chen, K. Tanaka, H. O. Wang, A Polynomial Observer Design for a Wider Class of Polynomial Fuzzy Systems, 2011 International Conference on Fuzzy Systems, Taipei, 2011 (Accepted).

[14] S. Prajna, A. Papachristodoulou, P. Seiler and P. A. Parrilo: SOSTOOLS:Sum of Squares Optimization Toolbox for MATLAB, Version 2.00, 2004

[15] T. Takagi and M. Sugeno, "Fuzzy Identification of Systems and Its Applications to Modeling and Control", IEEE Trans. on SMC 15, no. 1, pp.116-132, 1985.

[16] K. Tanaka, T. Ikeda, H. O. Wang, "Fuzzy Regulators and Fuzzy Observers: Relaxed Stability Conditions and LMI-Based Designs", IEEE Transactions on fuzzy systems, VOL. 6, NO. 2, pp.250-265, 1998

[17] J. F. Sturm: "Using SeDUMi 1.02, a MATLAB toolbox for optimization over symmetric cones", Optimization Methods and Software, vol. 11 \& 12, pp.625-653, August 1999.

[18] K. C. Toh, R. H. Tutuncu and M. J. Todd, "On the implement of SDPT3 (version 3.1) - A MATLAB software package for semidefinitequadratic-linear programming", 2004 IEEE International Conference on Computer Aided Control System Designs, pp.290-296, Sept. 2004. 